\title{
Hydroxyalkylierung, Acylierung, Formylierung und Carboxylierung von 2-Nitro- und 2-Chlor-1-(trimethylsilyl)benzol ${ }^{1)}$
}

\author{
Franz Effenberger* und Wolfgang Spiegler ${ }^{2)}$ \\ Institut für Organische Chemie der Universität Stuttgart, \\ Pfaffenwaldring 55, D-7000 Stuttgart 80
}

Eingegangen am 13. November 1984

Die präparative Anwendung der basekatalysierten Carbodesilylierung von Aryltrimethylsilanen wird an den Umsetzungen von 2-Nitro- (la) und 2-Chlor-1-(trimethylsilyl)benzol (1 b) mit Aldehyden, Ketonen, Carbonsäurefluoriden bzw. -anhydriden, Dimethylformamid und Kohlendioxid beschrieben. In guten Ausbeuten werden dabei die entsprechenden [(Trimethylsiloxy)alkyl]benzole 3 und 8, (Hydroxyalkyl)benzole 4,6 und 9, die Benzophenone 12, die Benzaldehyde 14 sowie die Benzoesäuren 17 erhalten. Die neue Methode ist eine nützliche Alternative zur elektrophilen Substitution sowie der Verwendung metallorganischer Verbindungen bei der Darstellung mehrfach substituierter Aromaten.

\section{Hydroxyalkylation, Acylation, Formylation, and Carboxylation of 2-Nitro- and 2-Chloro-1- (trimethylsilyl)benzene ${ }^{1)}$}

The synthetic application of base-catalyzed carbodesilylation of aryltrimethylsilanes is demonstrated in the reactions of 2-nitro- (1 a) and 2-chloro-1-(trimethylsilyl)benzene (1 b) with aldehydes, ketones, acyl fluorides and carboxylic anhydrides, respectively, dimethylformamide, and carbon dioxide. The corresponding [(trimethylsiloxy)alkyl]benzenes $\mathbf{3}$ and $\mathbf{8}$, (hydroxyalkyl)benzenes 4, 6, and 9, the benzophenones 12, the benzaldehydes 14, and the benzoic acids 17 are obtained in good yields. The new method is a useful alternative to the normal electrophilic substitution or the application of organometallic compounds, respectively, for the synthesis of polysubstituted benzenes.

In der vorstehenden Arbeit haben wir über die prinzipiellen Möglichkeiten der durch Basen katalysierten Carbodesilylierung von Aryl- bzw. Heteroaryltrimethylsilanen mit Benzaldehyd berichtet ${ }^{1)}$. Der unterschiedliche Einfluß verschiedener Basen auf die Reaktionsgeschwindigkeit und deren Abhängigkeit von der Stabilität der als Zwischenstufen postulierten Aryl- bzw. Heteroaryl-Anionen konnte aufgezeigt und gedeutet werden ${ }^{1}$. In der vorliegenden Arbeit wird nun auf die präparative Anwendung dieses Reaktionstyps zur Darstellung mehrfach substituierter Benzolderivate eingegangen.

Die direkte Hydroxyalkylierung, Acylierung, Formylierung oder Carboxylierung von Acceptor-substituierten Aromaten wie z. B. Nitrobenzol mittels Friedel-Crafts-Reaktionen ist im allgemeinen nicht möglich; einerseits reicht das elektrophile Potential der elektrophilen Agentien in diesen Fällen nicht aus, andererseits bilden die Acceptor-substituierten Aromaten ( $\mathrm{R}=\mathrm{NO}_{2}, \mathrm{COR}, \mathrm{CN}$ etc.) mit den FC-Katalysatoren Komplexe, die einen elektrophilen Angriff an den Aromaten noch stärker erschweren ${ }^{3)}$. In der Regel werden deshalb die entsprechenden metallorganischen Verbindungen (Grignard- oder Lithium-Verbindun- 
gen) hergestellt und mit Elektrophilen umgesetzt. Hierbei erfolgen jedoch häufig Nebenreaktionen, wie z.B. eine Weiterreaktion der bei der Acylierung gebildeten Ketone zu tertiären Alkoholen, eine Reaktion der metallorganischen Verbindungen mit funktionellen Gruppen ( $\mathrm{NO}_{2}, \mathrm{CN}$ etc. $)^{4)}$ oder bei den ortho-Halogenmetallorganylen eine Eliminierung zu den Arinen ${ }^{5)}$. Nur bei sehr niedrigen Reaktionstemperaturen (in der Regel $<-100^{\circ} \mathrm{C}$ ) lassen sich manche der angeführten Folgereaktionen vermeiden ${ }^{6)}$.

Da bei den basisch katalysierten Carbodesilylierungen die als Zwischenstufen postulierten Aryl-Anionen im Vergleich zu den aus den Grignard- oder Lithiumverbindungen entstehenden Spezies offensichtlich weniger frei vorliegen ${ }^{1)}$, sollten bei der Carbodesilylierung die unerwünschten Folgereaktionen nur in untergeordnetem Maße auftreten. Gerade bei Acceptor-substituierten Aromaten sollte deshalb die basisch katalysierte Carbodesilylierung zur Synthese mehrfach substituierter Aromaten eine nützliche präparative Ergänzung zu der Darstellung ausgehend von metallorganischen Verbindungen sein. Diese Annahmen konnten wir in vollem Umfang durch die nachstehend beschriebenen Umsetzungen von Nitround Chlor-substituierten (Trimethylsilyl)benzolen mit Aldehyden, Ketonen, Carbonsäurederivaten, Dimethylformamid und Kohlendioxid bestätigen.

\section{Hydroxyalkylierungen mit Aldehyden und Ketonen}

Die Umsetzungen von 2-Nitro- (1 a) und 2-Chlor-1-(trimethylsilyl)benzol (1 b) mit den Benzaldehyden 2a-f führten in Dimethylformamid (DMF) als Lösungsmittel in Gegenwart der Katalysatoren Kalium-tert-butylat $\left(\mathrm{Me}_{3} \mathrm{COK}\right)$ bei $20^{\circ} \mathrm{C}$ oder Kaliumfluorid bei $100^{\circ} \mathrm{C}$ erwartungsgemäß zu den Trimethylsilylethern 3 bzw. den Benzhydrolen 4 (Tab. 1).
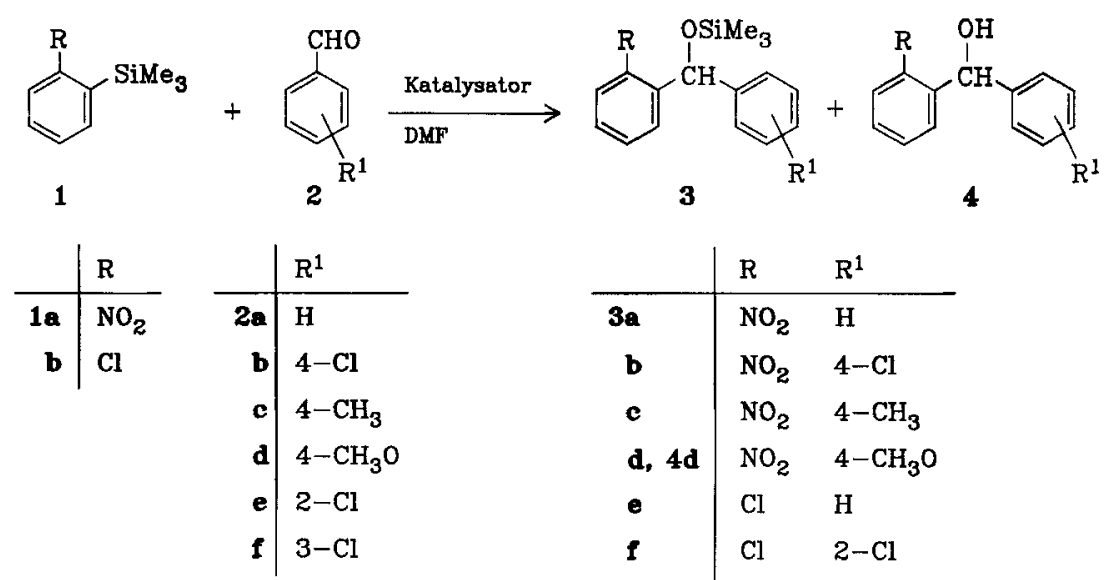

\begin{tabular}{c|ll} 
& $\mathrm{R}$ & $\mathrm{R}^{1}$ \\
\hline $\mathbf{3 a}$ & $\mathrm{NO}_{2}$ & $\mathrm{H}$ \\
$\mathbf{b}$ & $\mathrm{NO}_{2}$ & $4-\mathrm{Cl}$ \\
$\mathbf{c}$ & $\mathrm{NO}_{2}$ & $4-\mathrm{CH}_{3}$ \\
$\mathbf{d}, 4 \mathbf{4}$ & $\mathrm{NO}_{2}$ & $4-\mathrm{CH}_{3} \mathrm{O}$ \\
$\mathbf{e}$ & $\mathrm{Cl}$ & $\mathrm{H}$ \\
$\mathbf{f}$ & $\mathrm{Cl}$ & $2-\mathrm{Cl}$ \\
$\mathbf{g}$ & $\mathrm{Cl}$ & $3-\mathrm{Cl}$ \\
$\mathbf{h}$ & $\mathrm{Cl}$ & $4-\mathrm{Cl}$ \\
$\mathbf{i}, 4 \mathbf{1}$ & $\mathrm{Cl}$ & $4-\mathrm{CH}_{3}$ \\
$\mathbf{j}$ & $\mathrm{Cl}$ & $4-\mathrm{CH}_{3} \mathrm{O}$
\end{tabular}

Die aliphatischen Aldehyde 5a,b ließen sich mit $\mathbf{1 a}, \mathbf{b}$ nur mit Tetraethylammoniumfluorid $\left(\mathrm{Et}_{4} \mathrm{NF} \cdot 2 \mathrm{H}_{2} \mathrm{O}\right)$ bei $20^{\circ} \mathrm{C}$ oder mit $\mathrm{KF}$ bei $80^{\circ} \mathrm{C}$ in Hexamethyl- 
Tab. 1. Basisch katalysierte Carbodesilylierung von 2-Nitro- (1a) und 2-Chlor-1-(trimethylsilyl)benzol (1 b) mit substituierten Benzaldehyden 2 in DMF und mit Acet- (5a) bzw. $n$-Butyraldehyd (5b) in HMPT

\begin{tabular}{|c|c|c|c|c|c|c|c|}
\hline 1 & $\begin{array}{l}\text { Alde- } \\
\text { hyd }\end{array}$ & $\begin{array}{l}\text { Kataly- } \\
\text { sator } \\
\text { (mol-\%) }\end{array}$ & $\begin{array}{l}\text { Rea } \\
\text { zeit } \\
\text { (h) }\end{array}$ & $\begin{array}{l}\text { tions- } \\
\text { temp. } \\
\left({ }^{\circ} \mathrm{C}\right)\end{array}$ & & Produkt & $\underset{(\%)}{\text { Ausb. }}$ \\
\hline $1 \mathbf{a}$ & $2 \mathbf{a}$ & $\begin{array}{l}\mathrm{Me}_{3} \mathrm{COK} \\
(10)\end{array}$ & 1 & -60 & $\mathbf{3 a}$ & \multirow[t]{2}{*}{$\begin{array}{l}\text { 2-Nitro-1-[phenyl(trime- } \\
\text { thylsiloxy)methyl]benzol }\end{array}$} & $92^{a, 1)}$ \\
\hline $1 \mathbf{a}$ & $2 \mathbf{a}$ & $\begin{array}{l}\mathrm{Me}_{3} \mathrm{COK} \\
(3.7)\end{array}$ & 1 & 20 & $3 \mathbf{a}$ & & $80^{b, 1)}$ \\
\hline $1 \mathbf{a}$ & $2 b$ & KF & 10 & 100 & $\mathbf{3 b}$ & \multirow[t]{2}{*}{$\begin{array}{l}\text { 1-[(4-Chlorphenyl)(trime- } \\
\text { thylsiloxy)methyl }]-2 \text {-nitro- } \\
\text { benzol }\end{array}$} & $74^{\text {a) }}$ \\
\hline $1 \mathbf{a}$ & $2 b$ & $\begin{array}{l}\text { KF } \\
(16)\end{array}$ & 12 & 100 & $\mathbf{3 b}$ & & $37^{\text {a) }}$ \\
\hline $1 \mathbf{a}$ & $2 c$ & $\mathrm{KF}$ & 2 & 100 & $3 \mathrm{c}$ & \multirow[t]{2}{*}{$\begin{array}{l}\text { 2-Nitro-1-[p-tolyl(trime- } \\
\text { thylsiloxy)methyl]benzol }\end{array}$} & $47^{\text {a) }}$ \\
\hline $1 \mathbf{a}$ & $2 \mathrm{c}$ & $\begin{array}{l}\mathrm{KF} \\
(20)\end{array}$ & 6 & 100 & $3 c$ & & $50^{\mathrm{b})}$ \\
\hline \multirow[t]{2}{*}{$1 \mathbf{a}$} & $2 d$ & $\begin{array}{l}\mathrm{Me}_{3} \mathrm{COK} \\
(15)\end{array}$ & 4 & 20 & 3d & $\begin{array}{l}\text { 1-[(4-Methoxyphenyl)(tri- } \\
\text { methylsiloxy)methyl]-2-ni- } \\
\text { trobenzol }\end{array}$ & $42^{a\}}$ \\
\hline & & & & & $4 d$ & \multirow{2}{*}{$\begin{array}{l}\text { (4-Methoxyphenyl)(2-nitro- } \\
\text { phenyl)methanol }\end{array}$} & $20^{21}$ \\
\hline $1 \mathbf{a}$ & $2 d$ & $\begin{array}{l}\mathrm{Me}_{3} \mathrm{COK} \\
\text { (15) }\end{array}$ & 4 & 20 & $3 d$ & & $13^{\text {b) }}$ \\
\hline $1 \mathbf{b}$ & $2 \mathbf{a}$ & $\begin{array}{l}\mathrm{Me}_{3} \mathrm{COK} \\
(20)\end{array}$ & 1 & -30 & $3 \mathbf{e}$ & \multirow[t]{2}{*}{$\begin{array}{l}\text { 1-Chlor-2-[phenyl(trime- } \\
\text { thylsiloxy)methyl]benzol }\end{array}$} & $80^{a, 1)}$ \\
\hline $1 \mathrm{~b}$ & $2 a$ & $\mathrm{Me}_{3} \mathrm{COK}$ & 1 & 20 & $3 \mathbf{e}$ & & $74^{b, 1)}$ \\
\hline $1 \mathrm{~b}$ & $2 e$ & $\begin{array}{l}\mathrm{Me}_{3} \mathrm{COK} \\
(10)\end{array}$ & 2 & 20 & $3 f$ & $\begin{array}{l}\text { 1-Chlor-2-[(2-chlorphe- } \\
\text { nyl)(trimethylsiloxy)me- } \\
\text { thyl] benzol }\end{array}$ & $61^{b)}$ \\
\hline $\mathbf{l b}$ & $2 f$ & $\begin{array}{l}\mathrm{Me}_{3} \mathrm{COK} \\
(10)\end{array}$ & 2 & 20 & $3 \mathbf{g}$ & $\begin{array}{l}\text { 1-Chlor-2-[(3-chlorphe- } \\
\text { nyl)(trimethylsiloxy)me- } \\
\text { thyl]benzol }\end{array}$ & $58^{\text {b) }}$ \\
\hline $1 \mathrm{~b}$ & $2 \mathrm{~b}$ & $\begin{array}{l}\mathrm{Me}_{3} \mathrm{COK} \\
(10)\end{array}$ & 2 & 20 & $3 \mathbf{h}$ & $\begin{array}{l}\text { 1-Chlor-2-[(4-chlorphe- } \\
\text { nyl)(trimethylsiloxy)me- } \\
\text { thyl]benzol }\end{array}$ & $60^{\mathrm{b})}$ \\
\hline \multirow[t]{2}{*}{$1 \mathbf{b}$} & $2 c$ & $\begin{array}{l}\mathrm{Me}_{3} \mathrm{COK} \\
(23)\end{array}$ & 4 & 20 & $3 \mathbf{i}$ & $\begin{array}{l}\text { 1-Chlor-2-[(p-tolyl)(trime- } \\
\text { thylsiloxy)methyl]benzol }\end{array}$ & $11^{\text {a) }}$ \\
\hline & & & & + & $4 i$ & $\begin{array}{l}\text { (2-Chlorphenyl)-p-tolylme- } \\
\text { thanol }\end{array}$ & $48^{\text {a) }}$ \\
\hline $1 \mathrm{~b}$ & $2 d$ & $\begin{array}{l}\mathrm{Me}_{3} \mathrm{COK} \\
(33)\end{array}$ & 4 & 20 & $3 \mathbf{j}$ & $\begin{array}{l}\text { 1-Chlor-2-[(4-methoxyphe- } \\
\text { nyl)(trimethylsiloxy)me- } \\
\text { thyl]benzol }\end{array}$ & $52^{\text {a) }}$ \\
\hline $1 \mathrm{~b}$ & $2 d$ & $\begin{array}{l}\mathrm{Me}_{3} \mathrm{COK} \\
(30)\end{array}$ & 4 & 20 & $\mathbf{3 j}$ & & $38^{\mathrm{b})}$ \\
\hline $1 \mathbf{a}$ & $5 \mathbf{a}$ & $\begin{array}{l}\mathrm{Et}_{4} \mathrm{NF} \cdot 2 \mathrm{H}_{2} \mathrm{O} \\
(10)\end{array}$ & $1 / 2$ & $20^{c)}$ & $6 a$ & 1-(2-Nitrophenyl)ethanol & $74^{\mathrm{b})}$ \\
\hline $1 \mathbf{a}$ & $5 \mathbf{b}$ & $\begin{array}{l}\mathrm{KF} \\
(10)\end{array}$ & 10 & 80 & $6 b$ & 1-(2-Nitrophenyl)-1-butanol & $74^{\text {b) }}$ \\
\hline $1 \mathbf{b}$ & $5 \mathbf{a}$ & $\begin{array}{l}\mathrm{Et}_{4} \mathrm{NF} \cdot 2 \mathrm{H}_{2} \mathrm{O} \\
(10)\end{array}$ & 12 & $20^{c)}$ & $6 c$ & 1-(2-Chlorphenyl)ethanol & $36^{\text {b) }}$ \\
\hline $1 \mathrm{~b}$ & $5 \mathbf{b}$ & $\begin{array}{l}\mathrm{KF} \\
(10)\end{array}$ & 12 & 80 & $6 d$ & $\begin{array}{l}\text { 1-(2-Chlorphenyl)-1-buta- } \\
\text { nol }\end{array}$ & $46^{b)}$ \\
\hline
\end{tabular}

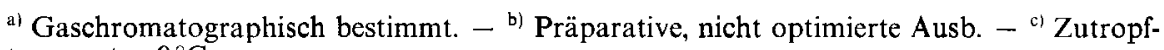
temperatur $0{ }^{\circ} \mathrm{C}$. 
phosphorsäuretriamid (HMPT) umsetzen, da in Gegenwart von $\mathrm{Me}_{3} \mathrm{COK}$ als Katalysator bevorzugt Aldolreaktion erfolgte. Nach wäßriger Aufarbeitung wurden als Reaktionsprodukte die (Hydroxyalkyl)benzole 6 isoliert (Tab. 1).

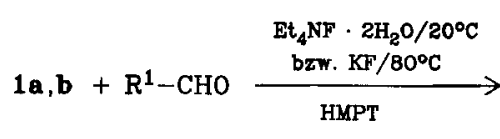

5

$$
\begin{array}{l|ll} 
& \mathbf{5 a} & \mathbf{b} \\
\hline \mathrm{R}^{1} & \mathrm{CH}_{3} & \mathrm{CH}_{3} \mathrm{CH}_{2} \mathrm{CH}_{2}
\end{array}
$$

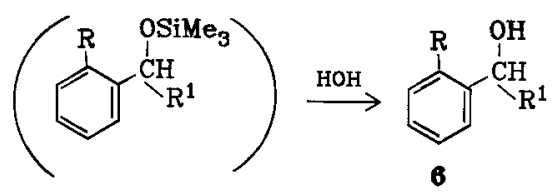

\begin{tabular}{l|llll} 
& $\mathbf{6 a}$ & $\mathbf{b}$ & $\mathbf{c}$ & $\mathbf{d}$ \\
\hline $\mathrm{R}$ & $\mathrm{NO}_{2}$ & $\mathrm{NO}_{2}$ & $\mathrm{Cl}$ & $\mathrm{Cl}$ \\
$\mathrm{R}^{1}$ & $\mathrm{CH}_{3}$ & $\mathrm{CH}_{3} \mathrm{CH}_{2} \mathrm{CH}_{2}$ & $\mathrm{CH}_{3}$ & $\mathrm{CH}_{3} \mathrm{CH}_{2} \mathrm{CH}_{2}$
\end{tabular}

Versuche zur Hydroxylierung von 1a,b mit Formaldehyd und Chloral verliefen negativ. Die Wechselwirkung dieser Aldehyde mit dem Katalysator (Addition an die Carbonylgruppe) ist offensichtlich gegenüber der Addition an das Silicium stark bevorzugt und führt zu irreversiblen Folgereaktionen, z. B. Polymerisation des Formaldehyds bzw. der bekannten $\mathrm{C}-\mathrm{C}$-Bindungsspaltung beim Chloral unter Bildung von Chloroform und Formiat.

Von den aus sterischen und elektronischen Gründen im Vergleich zu den Aldehyden deutlich weniger carbonylaktiven Ketonen ${ }^{7)}$ setzten wir Benzophenon (7a), Aceton (7b) und Acetophenon (7c) mit den Arylsilanen 1a,b unter Basekatalyse in HMPT als Lösungsmittel um. Die zu erwartenden Carbodesilylierungsprodukte 8 bzw. 9 wurden dabei je nach eingesetztem Keton in unterschiedlichen Ausbeuten erhalten (Tab. 2).

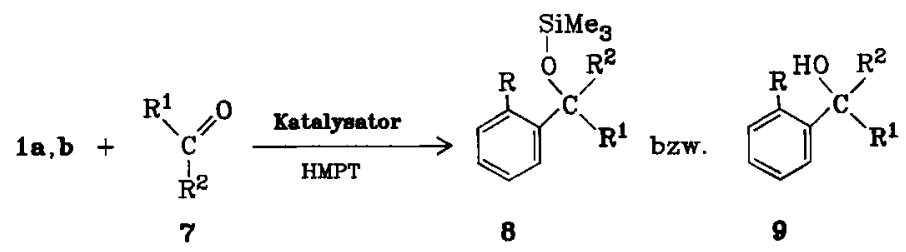

\begin{tabular}{l|llll} 
& $\mathbf{7 a}$ & $\mathbf{b}$ & $\mathbf{c}$ & $\mathbf{d}$ \\
\hline $\mathrm{R}^{1}$ & $\mathrm{Ph}$ & $\mathrm{CH}_{3}$ & $\mathrm{Ph}$ & \\
$\mathrm{R}^{2}$ & $\mathrm{Ph}$ & $\mathrm{CH}_{3}$ & $\mathrm{CH}_{3}$ & {$\left[{ }_{1}^{\mathrm{C}} \mathrm{CH}_{2}\right]_{5}$}
\end{tabular}

\begin{tabular}{l|lllllll} 
& $\mathbf{8 a}$ & $\mathbf{9 b}$ & $\mathbf{8 c}$ & $\mathbf{8 d}$ & $\mathbf{8 e}$ & $\mathbf{9 f}$ & $\mathbf{9 g}$ \\
\hline $\mathrm{R}$ & $\mathrm{NO}_{2}$ & $\mathrm{NO}_{2}$ & $\mathrm{NO}_{2}$ & $\mathrm{NO}_{2}$ & $\mathrm{Cl}$ & $\mathrm{Cl}$ & $\mathrm{Cl}$ \\
$\mathrm{R}^{1}$ & $\mathrm{Ph}$ & $\mathrm{CH}_{3}$ & $\mathrm{Ph}$ & {$\left[\mathrm{CH}_{2}\right]_{5}$} & $\mathrm{Ph}$ & $\mathrm{CH}_{3}$ & $\mathrm{Ph}$ \\
$\mathrm{R}^{2}$ & $\mathrm{Ph}$ & $\mathrm{CH}_{3}$ & $\mathrm{CH}_{3}$ & ${ }_{1}$ & $\mathrm{Ph}$ & $\mathrm{CH}_{3}$ & $\mathrm{CH}_{3}$
\end{tabular}

Niedrige Ausbeuten resultierten vor allem mit dem im Vergleich zu Aceton (7b) stärker enolisierten Acetophenon (7c) ${ }^{8}$. Im Falle der Umsetzungen von 1a mit 7c haben wir sowohl nach wäßriger als auch nach nicht-wäßriger Aufarbeitung neben kleinen Mengen Carbodesilylierungsprodukt 8c noch Nitrobenzol als Protodesilylierungsprodukt in Ausbeuten von $68-92 \%$ nachgewiesen sowie bei wäßriger Aufarbeitung ca. $80 \%$ des eingesetzten Acetophenons (s. exp. Teil). Diese

Chem. Ber. 118 (1985) 
Tab. 2. Basisch katalysierte Carbodesilylierung von 2-Nitro- (1 a) und 2-Chlor-1-(trimethylsilyl)benzol (1 b) mit Ketonen 7 in HMPT

\begin{tabular}{|c|c|c|c|c|c|c|c|}
\hline 1 & $\begin{array}{l}\text { Ke- } \\
\text { ton }\end{array}$ & $\begin{array}{l}\text { Kataly- } \\
\text { sator } \\
\text { (mol- \%) }\end{array}$ & $\begin{array}{l}\text { Reak } \\
\text { zeit } \\
\text { (h) }\end{array}$ & $\begin{array}{l}\text { tions- } \\
\text { temp. } \\
\left({ }^{\circ} \mathrm{C}\right)\end{array}$ & & Produkt & $\begin{array}{c}\text { Ausb. } \\
(\%)\end{array}$ \\
\hline $1 \mathbf{a}$ & $7 \mathbf{a}$ & $\begin{array}{l}\text { KF } \\
(10)\end{array}$ & 48 & 80 & $8 \mathbf{a}$ & \multirow{2}{*}{$\begin{array}{l}\text { (2-Nitrophenyl)diphenyl(tri- } \\
\text { methylsiloxy)methan }\end{array}$} & $52^{\text {a) }}$ \\
\hline $1 \mathrm{a}$ & $7 \mathbf{a}$ & $\begin{array}{l}\mathrm{Me}_{3} \mathrm{COK} \\
\text { (10) }\end{array}$ & $\begin{array}{r}2 \\
+3\end{array}$ & $\begin{array}{r}20 \\
100\end{array}$ & $8 \mathbf{a}$ & & $61^{\text {b) }}$ \\
\hline $1 \mathrm{a}$ & $7 b$ & $\begin{array}{l}\mathrm{Me}_{3} \mathrm{COK} \\
(20)\end{array}$ & & 20 & $9 b$ & \multirow[t]{2}{*}{$\begin{array}{l}\text { 2-(2-Nitrophenyl)-2-propa- } \\
\text { nol }\end{array}$} & $62^{\text {a) }}$ \\
\hline $1 \mathrm{a}$ & $7 b$ & $\begin{array}{l}\mathrm{Me}_{3} \mathrm{COK} \\
(10)\end{array}$ & 1 & 20 & $9 \mathrm{~b}$ & & $56^{\text {b) }}$ \\
\hline $1 \mathrm{a}$ & $7 c$ & $\begin{array}{l}\mathrm{CsF} \\
(25)\end{array}$ & 24 & 20 & $8 c$ & \multirow[t]{2}{*}{$\begin{array}{l}\text { 1-(2-Nitrophenyl)-1-phenyl- } \\
1 \text {-(trimethylsiloxy)ethan }\end{array}$} & $11^{\mathrm{a})}$ \\
\hline $1 \mathbf{a}$ & $7 c$ & $\begin{array}{l}\mathrm{Et}_{4} \mathrm{NF} \cdot 2 \mathrm{H}_{2} \mathrm{O} \\
(10)\end{array}$ & 1 & 20 & $8 \mathrm{c}$ & & $13^{\text {b) }}$ \\
\hline $1 \mathrm{a}$ & $7 d$ & $\begin{array}{l}\text { KF } \\
(10)\end{array}$ & 48 & 80 & $8 d$ & \multirow{3}{*}{$\begin{array}{l}\text { 1-(2-Nitrophenyl)-1-(trime- } \\
\text { thylsiloxy)cyclohexan } \\
\text { (2-Chlorphenyl)diphenyl- } \\
\text { (trimethylsiloxy)methan }\end{array}$} & $17^{\mathrm{b})}$ \\
\hline $1 \mathrm{~b}$ & $7 \mathbf{a}$ & $\begin{array}{l}\text { KF } \\
(10)\end{array}$ & 39 & 80 & $8 \mathbf{e}$ & & $39^{\text {a) }}$ \\
\hline $1 \mathrm{~b}$ & $7 \mathbf{a}$ & $\begin{array}{l}\mathrm{Me}_{3} \mathrm{COK} \\
(35)\end{array}$ & 2 & 20 & $8 \mathbf{e}$ & & $46^{\text {b) }}$ \\
\hline $1 \mathrm{~b}$ & $7 b$ & $\begin{array}{l}\mathrm{Et}_{4} \mathrm{NF} \cdot 2 \mathrm{H}_{2} \mathrm{O} \\
(30)\end{array}$ & 1 & 20 & 9f & \multirow{2}{*}{$\begin{array}{l}\text { 2-(2-Chlorphenyl)-2-propa- } \\
\text { nol } \\
\text { 1-(2-Chlorphenyl)-1-phenyl- } \\
\text { ethanol }\end{array}$} & $28^{\mathrm{b})}$ \\
\hline lb & $7 c$ & $\begin{array}{l}\mathrm{Et}_{4} \mathrm{NF} \cdot 2 \mathrm{H}_{2} \mathrm{O} \\
(40)\end{array}$ & 2 & 20 & $9 \mathrm{~g}$ & & $8^{\text {b) }}$ \\
\hline
\end{tabular}

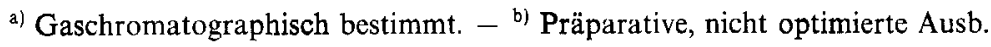

Befunde sind mit der größeren Acidität des Acetophenons zu erklären. Durch das Fluorid erfolgt bevorzugt eine Spaltung der Aryl-Silicium-Bindung unter Bildung des Aryl-Anions $\mathbf{A}$ und $\mathrm{Me}_{3} \mathrm{SiF}$. Das Anion A reagiert dann mit Acetophenon (7c) zu Nitrobenzol und dem Enolat B weiter, letzteres ergibt mit dem gebildeten $\mathrm{Me}_{3} \mathrm{SiF}$ den Trimethylsilylether $\mathbf{C}$ unter Rückbildung des Fluorids. Bei wäßriger<smiles>CSc1ccccc1[N+](=O)[O-]</smiles>

$1 \mathbf{a}$

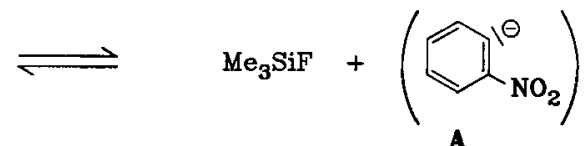

A<smiles>C=[V]</smiles><smiles>CC(=O)c1ccccc1</smiles>

Chem. Ber. 118 (1985) 
Aufarbeitung bildet sich aus $\mathbf{C}$ Acetophenon (7c) zurück, das - wie oben schon erwähnt - in hoher Ausbeute zurückgewonnen wurde.

Die in der Literatur beschriebene basekatalysierte Umsetzung von (Trimethylsilyl)pentafluorbenzol mit Acetophenon (7c) ergibt in analoger Weise $81 \%$ (1-Phenylvinyl)(trimethylsilyl)ether"), was als Bestätigung unserer Vorstellungen gewertet werden kann.

\section{Acylierungen mit Carbonsäurederivaten}

Die basekatalysierte Acylierung der Silylaromaten 1 a, b haben wir mit Carbonsäure-fluoriden, -chloriden und -anhydriden untersucht. Gute Ausbeuten an aromatischen Ketonen 12 wurden lediglich mit Benzoylfluorid (10a) in Gegenwart von $\mathrm{KF}$ erhalten. Mit Acetylfluorid in Gegenwart von Cäsiumfluorid als Katalysator entstanden aus 1 a nur ca. $10 \%$ des Carbodesilylierungsproduktes Methyl(2-nitrophenyl)keton. Alle Versuche, anstelle der Säurefluoride die leichter zugänglichen Carbonsäurechloride als Acylierungsmittel einzusetzen, blieben erfolglos. Selbst bei Verwendung molarer Mengen Kaliumfluorid, das mit dem Säurechlorid das Halogen austauschen könnte ${ }^{10}$, wurde kein Acylierungsprodukt erhalten. Benzoesäureanhydrid (10b) ergab mit 1a und Kaliumbenzoat als Katalysator 2-Nitrobenzophenon(12a) mit 32\% Ausbeute, während mit Acetanhydrid auch bei großer Variation der Reaktionsbedingungen (Lösungsmittel, Katalysator) kein Acylierungsprodukt erhalten wurde.

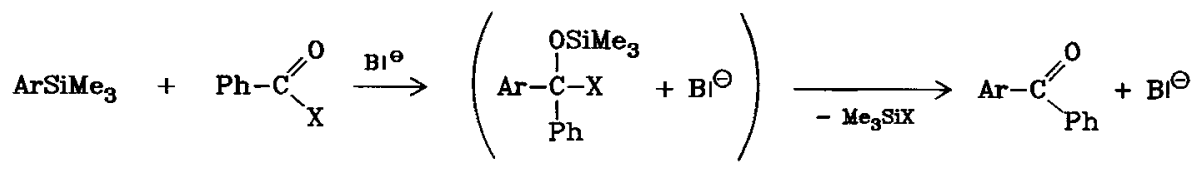
1a, b 10a, b

11 $12 \mathrm{a}, \mathrm{b}$ \begin{tabular}{l|ll} 
& $10 \mathbf{a}$ & $\mathrm{b}$ \\
\hline $\mathrm{X}$ & $\mathrm{F}$ & OCOPh
\end{tabular}

\begin{tabular}{l|ll} 
& $1 \mathrm{a}, 12 \mathrm{a}$ & $1 \mathrm{~b}, 12 \mathrm{~b}$ \\
\hline $\mathrm{Ar}$ & $2-\mathrm{NO}_{2} \mathrm{C}_{8} \mathrm{H}_{4}$ & $2-\mathrm{ClC}_{6} \mathrm{H}_{4}$
\end{tabular}

$1 \mathrm{a}+12 \mathrm{a} \underset{24 \mathrm{~h} / 100^{\circ} \mathrm{C}}{\stackrel{\mathrm{KF} / \mathrm{MMPT}}{\longrightarrow}}$

13a

$13 b$

Es ist anzunehmen, daß bei der Acylierung die tetraedrische Zwischenstufe 11 durchlaufen wird, die sehr leicht $\mathrm{Me}_{3} \mathrm{SiX}$ unter Bildung von 12 abspaltet. Folgereaktionen der Ketone $\mathbf{1 2}$ haben wir bei den vorstehend beschriebenen Umsetzungen nicht beobachtet. Lediglich bei einer getrennten Umsetzung von 1a mit 2-Nitrobenzophenon (12a) in Gegenwart von KF unter drastischeren Reaktionsbedingungen $\left(24 \mathrm{~h}\right.$ Erhitzen in HMPT bei $\left.100^{\circ} \mathrm{C}\right)$ erhielten wir Bis(2-nitrophenyl)phenylmethanol (13a) und 2-(2-Nitrophenyl)benzophenon (13b) in jeweils 6proz. Ausbeute. Die Bildung von 13a ist über eine weitere Carbodesilylierung

Chem. Ber, 118 (1985) 
von $1 \mathrm{a}$ durch $12 \mathrm{a}$ zu verstehen, die von $13 \mathrm{~b}$ über eine nucleophile Substitution der $\mathrm{NO}_{2}$-Gruppe in $12 \mathrm{a}$ durch das aus $1 \mathfrak{a}$ und $\mathrm{KF}$ gebildete 2-Nitrophenyl-Anion.

\section{Formylierung mit Dimethylformamid}

Dimethylformamid (DMF), das von uns sehr häufig eingesetzte Lösungsmittel bei basekatalysierten Carbodesilylierungsreaktionen (s. oben und Lit. ${ }^{1}$ ), kann selbst als Elektrophil z.B. mit Grignardverbindungen unter Bildung von Aldehyden reagieren ${ }^{11}$. Bei der Zugabe von Basen zu Lösungen von Aryltrimethylsilanen in DMF unter Ausschluß anderer Elektrophile war deshalb durchaus mit einer Formylierung der Aromaten zu rechnen.

Setzt man 1a in DMF mit $\mathrm{Me}_{3} \mathrm{COK}$ als Katalysator um, so entsteht 2-Nitrobenzaldehyd (14), der als 2,4-Dinitrophenylhydrazon isoliert wurde. Die Ausbeute an 14 hängt in starkem $\mathrm{Maße}$ von der zugesetzten Menge $\mathrm{Me}_{3} \mathrm{COK}$ ab, optimale Ausbeuten werden mit ca. $10 \mathrm{~mol}-\% \mathrm{Me}_{3} \mathrm{COK}$ erhalten. Mit größeren Mengen an Base und längeren Reaktionszeiten nimmt der Anteil an 14 unter Bildung von Nitrobenzol wieder ab. Diesen Befund deuten wir über die Spaltung des gebildeten Aldehyds 14 durch das tert-Butylat.

$$
1 \mathbf{a}+\mathrm{DMF} \underset{-\mathrm{Be}_{3} \mathrm{SiNMe}_{2}}{\stackrel{\mathrm{B}^{\ominus}}{\longrightarrow}}
$$<smiles>O=Cc1ccccc1[N+](=O)[O-]</smiles>

14
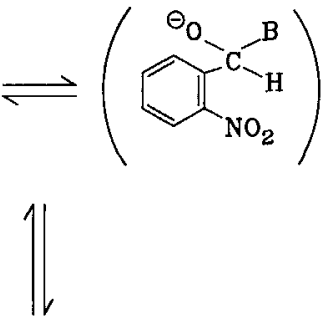

$\mathrm{B}^{\ominus}=\mathrm{Me}_{3} \mathrm{CO}{ }^{\ominus}$<smiles>O=C[18CH2]O</smiles>

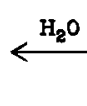<smiles>O=[N+]([O-])c1ccccc1</smiles><smiles>CCCCC[Pb]C=O</smiles>

\section{Carboxylierung mit Kohlendioxid}

Die Umsetzung metallorganischer Verbindungen mit Kohlendioxid dient häufig zur Darstellung von Carbonsäuren. Trotz seines niedrigen elektrophilen Potentials reagiert Kohlendioxid basekatalysiert mit Arylsilanen unter Carbodesilylierung. So konnten 1a und b in HMPT mit Kohlendioxid unter Druck und in Gegenwart molarer Mengen Kalium- bzw. Cäsiumfluorid in guten Ausbeuten zu den entsprechenden Benzoesäuren 17 umgesetzt werden.

Mit nur 10 mol-\% KF erfolgte mit 1 a unter diesen Bedingungen nur ein 10proz. Umsatz zu 2-Nitrobenzoesäure (17a). Offensichtlich wird der bei der Reaktion evtl. gebildete Silylester 16a mit KF leichter zum entsprechenden Benzoat 15a gespalten als das Arylsilan $1 \mathrm{a}$ zum entsprechenden instabileren Aryl-Anion. Das Benzoat 15a seinerseits ist als basischer Katalysator für die Carboxylierungsreaktion wohl zu wenig aktiv. 


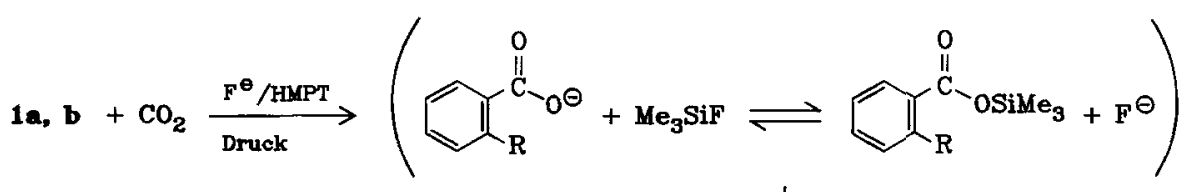

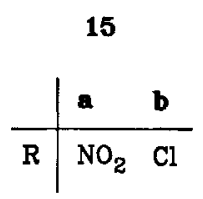

$\downarrow^{\mathrm{H}_{2} \mathrm{O}} \quad 16$

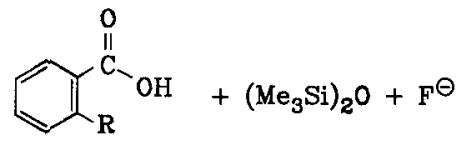

Zusammenfassend ist festzustellen, daß die durch Basen katalysierte Carbodesilylierung von Aryltrimethylsilanen zur Hydroxyalkylierung, Acylierung, Formylierung und Carboxylierung von Acceptor-substituierten Aromaten gut geeignet ist, und daß sie eine nützliche Ergänzung zu den normalen Friedel-CraftsReaktionen einerseits und den Umsetzungen metallorganischer Verbindungen mit Elektrophilen andererseits darstellt. Die an den Arylsilanen $\mathbf{1}$ a, b untersuchten Reaktionen lassen sich unter Berücksichtigung der in der vorstehenden Arbeit ${ }^{1)}$ beschriebenen Befunde mit Sicherheit auf viele andere Aryl- und Heteroaryltrimethylsilane übertragen. Eine interessante Anwendung dieses Reaktionstyps zur Darstellung mehrfach substituierter Benzole bzw. Anthrachinone wurde kürzlich von Mills und Snieckus ${ }^{12)}$ publiziert.

Wir danken dem Fonds der Chemischen Industrie und der Deutschen Forschungsgemeinschaft für die Unterstützung der Arbeit. Außerdem gilt unser Dank Herrn cand. chem. $W$. Ulmer für engagierte Mitarbeit im Rahmen des Forschungspraktikums.

\section{Experimenteller Teil}

Gaschromatographie: Gerät 5700 A der Fa. Hewlett-Packard mit Flammenionisationsdetektor (FID), Integrator: Spectraphysics Minigrator. Trägergas $30 \mathrm{ml} / \mathrm{min}$ Reinstickstoff. Glaskapillare $2.3 \mathrm{~m} \times 2 \mathrm{~mm}$. Stationäre Phase OV 101/5\% auf Gaschrom Q und OV 25/ $5 \%$ auf Gaschrom Q. Temperaturprogramm $100-300^{\circ} \mathrm{C}, 16^{\circ} \mathrm{C} / \mathrm{min}$. Die Identifizierung der Produkte erfolgte durch Koinjektion von Vergleichssubstanzen, die Ausbeuten wurden nach Einwaage einer definierten Menge an internem Standard (ca. $0.1 \mathrm{~g}$ Tetralin pro mmol Reaktand) und mit Hilfe von Eichmessungen erhalten ${ }^{13)}$ - Präparative Säulenchromatographie nach Lit. ${ }^{14)}$, Glassäulen, Typ C, $40 \times 4 \mathrm{~cm}$, gefüllt mit Kieselgel (Fa. Merck, 0.015-0.025 $\mathrm{mm}$ ), ca. 6600 theor. Böden, UV-Detektor: Spektrochrom (Fa. Gilson). - ${ }^{1}$ H-NMR-Spektren: Geräte T 60, EM 360 der Fa. Varian sowie HX 90 der Fa. Bruker.

Sämtliche Reaktionen unter Basekatalyse erfolgten unter Luft- und Feuchtigkeitsausschluß unter Stickstoff oder Argon. - Kalium-tert-butylat $\left(\mathrm{Me}_{3} \mathrm{COK}\right)^{15)}$ wurde frisch sublimiert, die anderen Katalysatoren wurden nach Trocknen über Phosphorpentoxid (bis zu $300^{\circ} \mathrm{C}$ ) fein suspendiert mittels eines Vibrorührers im Ultraschallbad eingesetzt. Tetraethylammoniumfluorid $\left(\mathrm{Et}_{4} \mathrm{NF} \cdot \mathbf{2} \mathrm{H}_{2} \mathrm{O}\right.$ ) wurde aus Tetraethylammonium-tetrafluoroborat nach Lit. ${ }^{16)}$ synthetisiert ${ }^{11}$. 
Allgemeine Arbeitsweise: Reaktionen, bei denen der Katalysator vorgelegt wird, werden in einem Einhalskolben, solche, bei denen der Katalysator zu den Edukten gegeben wird, in einem Zweihalskolben mit Feststoffdosierstück durchgeführt. Die Gefäße werden mit Magnetrührstäbchen und Katalysator fünfmal i. Vak. ausgeheizt und mit Stickstoff begast. Anschließend wird das Gasableitungsrohr mit Hahn unter Stickstoff oder Argon gegen ein Septum (rubber septum der Fa. Aldrich) ausgetauscht. Die Zugabe der Reaktanden und der Lösungsmittel sowie die Probeentnahmen erfolgen durch das Septum mit einer Spritze. Die Gemische werden die angegebene Zeit bei der angegebenen Temperatur gerührt und anschließend aufgearbeitet.

\section{Aufarbeitung}

Methode A: Der Katalysator wird über eine Vak.-Fritte abgesaugt, anschließend wird aus der klaren Lösung das Lösungsmittel im Wasserstrahlvakuum über eine Kolonne abdestilliert.

Methode B: Der nach Methode A erhaltene Rückstand wird im genannten Eluens aufgenommen und über eine Glassäule (30 cm lang, gefüllt mit $60 \mu$-Kieselgel der Fa. Merck) filtriert.

Methode C: Das Reaktionsgemisch wird mit Wasser oder verd. Säurc $2 \mathrm{~h}$ gerührt und mit Ether extrahiert. Der Etherextrakt wird über Magnesiumsulfat getrocknet und im Rotationsverdampfer eingeengt.

Methode GC/A: Die dem Reaktionsgemisch entnommene Probe wird mit der 20-30fachen Menge Ether verdünnt. Nach Stehenlassen unter Verschluß bis zum Absetzen des Niederschlags wird mittels einer Pipette die klare Lösung abgesaugt und gaschromatographisch bestimmt.

Methode GC/B: Die dem Reaktionsgemisch entnommene Probe wird mit der 3 fachen Menge 2proz. Salzsäure oder 5proz. Schwefelsäure hydrolysiert und mit Ether extrahiert. Aus der über Magnesiumsulfat getrockneten Etherphase werden Proben mit einer Pipette abgesaugt und gaschromatographisch untersucht.

Carbodesilylierung von 2-Nitro- (1 a) und 2-Chlor-1-(trimethylsilyl) benzol (1 b)

1. Mit aromatischen Aldehyden

a) Von $1 \mathrm{a}$ mit 4-Chlorbenzaldehyd (2b): 1) $0.195 \mathrm{~g}$ (1.00 mmol) $1 \mathrm{a}$ werden mit $0.141 \mathrm{~g}$ (1.00 mmol) $2 \mathrm{~b}$ und $0.0116 \mathrm{~g}(0.20 \mathrm{mmol}) \mathrm{KF}$ in $3 \mathrm{ml}$ DMF $10 \mathrm{~h}$ bei $100^{\circ} \mathrm{C}$ gerührt. Anschließend wird nach Methode GC/A aufgearbeitet. Ausb. 74\% 1-[(4-Chlorphenyl)(trimethylsiloxy)methyl/-2-nitrobenzol (3b).

2) $2.44 \mathrm{~g}(12.5 \mathrm{mmol}) 1 \mathrm{a}$ werden mit $1.41 \mathrm{~g}(10.0 \mathrm{mmol}) 2 \mathrm{~b}$ und $0.116 \mathrm{~g}(2.0 \mathrm{mmol}) \mathrm{KF}$ in $30 \mathrm{ml} \mathrm{DMF} 12 \mathrm{~h}$ bei $100^{\circ} \mathrm{C}$ gerührt. Nach Methode B wird mit Petrolether/Essigester (PE/EE) (9:1) aufgearbeitet. Das Eluat wird eingeengt und der Rückstand destilliert. Ausb. $1.55 \mathrm{~g}(37 \%) 3 \mathrm{~b}$, Sdp. $144^{\circ} \mathrm{C} / 2.5 \cdot 10^{-2}$ Torr. $-{ }^{1} \mathrm{H}-\mathrm{NMR}\left(\mathrm{CDCl}_{3}\right): \delta=0.07(\mathrm{~s}, 9 \mathrm{H}$, $\left.\mathrm{Si}\left(\mathrm{CH}_{3}\right)_{3}\right), 6.52$ (s, $\left.\mathrm{CH}\right), 7.28-7.98$ (m, 8 aromat. $\left.\mathrm{H}\right)$.

$$
\begin{array}{lllllll}
\mathrm{C}_{16} \mathrm{H}_{18} \mathrm{ClNO}_{3} \mathrm{Si} \text { (335.9) } & \text { Ber. C } 57.21 & \text { H } 5.40 & \mathrm{Cl} 10.55 & \mathrm{~N} 4.17 \\
& \text { Gef. C } 57.09 & \text { H } 5.49 & \mathrm{Cl} 10.44 & \mathrm{~N} 4.43
\end{array}
$$

b) Von $1 \mathrm{a}$ mit 4-Methylbenzaldehyd (2c): 1) $0.195 \mathrm{~g}$ (1.00 mmol) $1 \mathrm{a}$ werden mit $0.12 \mathrm{~g}(1.0$ mmol) $2 \mathrm{c}$ und $0.006 \mathrm{~g}(0.1 \mathrm{mmol}) \mathrm{KF}$ in $3 \mathrm{ml} \mathrm{DMF} 2 \mathrm{~h}$ bei $100^{\circ} \mathrm{C}$ gerührt. Aufarbeitung nach Methode GC/A liefert 47\% 2-Nitro-1-[p-tolyl(trimethylsiloxy) methyl Jbenzol (3c).

2) $2.73 \mathrm{~g}(14 \mathrm{mmol}) 1 \mathrm{a}$ werden mit $1.2 \mathrm{~g}(10 \mathrm{mmol}) 2 \mathrm{c}$ und $0.162 \mathrm{~g}(2.8 \mathrm{mmol}) \mathrm{KF}$ in 30 ml DMF 6 h bei $100^{\circ} \mathrm{C}$ gerührt. Es wird nach Methode B mit PE/EE (9:1) aufgearbeitet. 
Nach Mitteldrucksäulenchromatographie des Rohproduktes mit PE/EE (50:1) Ausb. $1.58 \mathrm{~g}$ $(50 \%) 3 \mathrm{c} .-{ }^{1} \mathrm{H}-\mathrm{NMR}\left(\mathrm{CDCl}_{3}\right): \delta=0.07\left(\mathrm{~s}, 9 \mathrm{H}, \mathrm{Si}\left(\mathrm{CH}_{3}\right)_{3}\right), 2.30\left(\mathrm{~s}, 3 \mathrm{H}, \mathrm{CH}_{3}\right), 6.52(\mathrm{~s}, 1 \mathrm{H}$, $\mathrm{CH}), 7.00-8.00(\mathrm{~m}, 8$ aromat. $\mathrm{H})$.

$$
\mathrm{C}_{17} \mathrm{H}_{21} \mathrm{NO}_{3} \mathrm{Si} \text { (315.4) Ber. C } 64.72 \text { H } 6.71 \mathrm{~N} 4.44 \text { Gef. C } 64.66 \text { H } 6.81 \quad \mathrm{~N} 4.50
$$

c) Von $1 \mathrm{a}$ mit 4-Methoxybenzaldehyd (2d): 1) $0.195 \mathrm{~g}$ (1.00 mmol) 1 a werden mit $0.136 \mathrm{~g}$ (1.00 mmol) $2 \mathrm{~d}$ und $0.016 \mathrm{~g}(0.15 \mathrm{mmol}) \mathrm{Me}_{3} \mathrm{COK}$ in $3 \mathrm{ml} \mathrm{DMF} 4 \mathrm{~h}$ bei $20^{\circ} \mathrm{C}$ gerührt. Aufarbeitung nach Methode GC/A liefert 42\% 1-/(4-Methoxyphenyl)(trimethylsiloxy methyl]-2-nitrobenzol (3d) und 20\% (4-Methoxyphenyl)(2-nitrophenyl)methanol (4d).

2) $1.95 \mathrm{~g}$ (10 mmol) $1 \mathrm{a}$ werden mil $1.36 \mathrm{~g}(10 \mathrm{mmol}) 2 \mathrm{~d}$ und $0.168 \mathrm{~g}(1.5 \mathrm{mmol}) \mathrm{Me}_{3} \mathrm{COK}$ in $30 \mathrm{ml} \mathrm{DMF} 4 \mathrm{~h}$ bei $20^{\circ} \mathrm{C}$ gerührt. Aufarbeitung nach Methode B mit PE/EE (9:1). Nach Mitteldrucksäulenchromatographie des Rohprodukts mit PE/EE (50:1) Ausb. $0.43 \mathrm{~g}(13 \%)$ 3d. $-{ }^{1} \mathrm{H}-\mathrm{NMR}\left(\mathrm{CDCl}_{3}\right): \delta=0.07\left(\mathrm{~s}, 9 \mathrm{H}, \mathrm{Si}\left(\mathrm{CH}_{3}\right)_{3}\right), 3.78\left(\mathrm{~s}, 3 \mathrm{H}, \mathrm{OCH}_{3}\right), 6.50(\mathrm{~s}, 1 \mathrm{H}, \mathrm{CH})$, $6.78-8.03$ (m, 8 aromat. $\mathrm{H}$ ).

\section{$\mathrm{C}_{17} \mathrm{H}_{21} \mathrm{NO}_{4} \mathrm{Si}$ (331.4) Ber. C 61.60 H 6.38 N 4.22 Gif, C 61.83 H 6.55 N 4.15}

d) Von $1 \mathrm{~b}$ mit 2-Chlor- (2e), 3-Chlor- (2f) und 4-Chlorbenzaldehyd (2b): $\mathrm{Zu}$ jeweils $3.69 \mathrm{~g}$ (20 mmol) 1 b und $2.81 \mathrm{~g} \mathrm{(20} \mathrm{mmol)} 2 \mathrm{e}$, f oder $\mathbf{b}$ in $20 \mathrm{ml}$ DMF werden bei Raumtemp. auf einmal $0.224 \mathrm{~g} \mathrm{(2.00} \mathrm{mmol)} \mathrm{Me}_{3} \mathrm{COK}$ gegeben. Nach $2 \mathrm{~h}$ Rühren und Aufarbeiten nach Methode A wird der Rückstand i.Vak. destilliert. Ausb. $3.96 \mathrm{~g}$ (61\%) 1-Chlor-2-/(2-chlorphenyl)(trimethylsiloxy)methylJbenzol (3f), Sdp. $102-104^{\circ} \mathrm{C} / 7 \cdot 10^{-3}$ Torr. $-{ }^{1} \mathrm{H}-\mathrm{NMR}$ $\left(\mathrm{CDCl}_{3}\right): \delta=0.13\left(\mathrm{~s}, 9 \mathrm{H}, \mathrm{Si}\left(\mathrm{CH}_{3}\right)_{3}\right), 6.75(\mathrm{~s}, \mathrm{CH}), 7.13-7.65(\mathrm{~m}, 8 \mathrm{H}, \mathrm{Ph})$.

$3.76 \mathrm{~g}(58 \%)$ 1-Chlor-2-( (3-chlorphenyl) (trimethylsiloxy)methyl]benzol $(3 \mathrm{~g}), \mathrm{Sdp} .118^{\circ} \mathrm{C} /$ $5 \cdot 10^{-2}$ Torr. $-{ }^{1} \mathrm{H}-\mathrm{NMR}\left(\mathrm{CDCl}_{3}\right): \delta=0.08\left(\mathrm{~s}, 9 \mathrm{H}, \mathrm{Si}\left(\mathrm{CH}_{3}\right)_{3}\right), 6.20(\mathrm{~s}, \mathrm{CH}), 7.07-7.71(\mathrm{~m}$, 8 aromat. $\mathrm{H})$.

$3.88 \mathrm{~g}(60 \%)$ 1-Chlor-2-[(4-chlorphenyl)(trimethylsiloxy)methyl]benzol (3h), Sdp. $112-114^{\circ} \mathrm{C} / 8 \cdot 10^{-2}$ Torr. $-{ }^{1} \mathrm{H}-\mathrm{NMR}\left(\mathrm{CDCl}_{3}\right): \delta=0.08$ (s, $\left.9 \mathrm{H}, \mathrm{Si}\left(\mathrm{CH}_{3}\right)_{3}\right), 6.12(\mathrm{~s}, \mathrm{CH})$, $7.07-7.90$ (m, 8 aromat. $\mathrm{H})$.

$$
\mathrm{C}_{16} \mathrm{H}_{18} \mathrm{Cl}_{2} \mathrm{OSi}(325.3) \quad \text { Ber. C } 59.07 \mathrm{H} 5.57 \mathrm{Cl} 21.79 \text { 3f: Gef. C } 58.88 \text { H } 5.40 \text { Cl } 21.85
$$

$3 \mathrm{~g}$ : Gef. C 58.96 H $5.65 \mathrm{Cl} 21.86$

3h: Gef. C 59.09 H 5.67 Cl 22.03

e) Von $1 \mathrm{~b}$ mit 4-Methylbenzaldehyd (2c): $3.12 \mathrm{~g}$ (17 mmol) $1 \mathrm{~b}$ werden mit $2.40 \mathrm{~g}$ (20 mmol) 2c und $0.448 \mathrm{~g}(4.00 \mathrm{mmol}) \mathrm{Me}_{3} \mathrm{COK}$ in $60 \mathrm{ml} \mathrm{DMF} 4 \mathrm{~h}$ bei $20{ }^{\circ} \mathrm{C}$ gerührt. Eine Probe wird nach Methode GC/A aufgearbeitet. Ausb. $11 \%$ 1-Chlor-2-(p-tolyl(trimethylsiloxy)methyl Jbenzol (3i) und 48\% (2-Chlorphenyl)-p-tolylmethanol (4i). Das Rcaktionsgemisch wird dann nach Methode C mit $180 \mathrm{ml}$ 5proz. Salzsäure aufgearbeitet. Der so erhaltene Rückstand wird durch Mitteldrucksäulenchromatographie zunächst mit PE/EE (50:1) gereinigt, wobei als 1. Fraktion $0.25 \mathrm{~g}$ einer farblosen Flüssigkeit (vermutlich 3i) resultieren. Mit reinem EE als Eluens werden anschlieBend $0.51 \mathrm{~g} 4 \mathbf{i}$ isoliert. Die 1. Fraktion wird mit methanolischer Salzsäure hydrolysiert und das Rohprodukt durch Mitteldrucksäulenchromatugraphie mit EE gereinigt. Ausb. $0.69 \mathrm{~g}(18 \%) 4 \mathrm{i}$, Schmp. $67^{\circ} \mathrm{C}$ (Lit. ${ }^{17)} 67^{\circ} \mathrm{C}$ ).

f) Von $1 \mathrm{~b}$ mit 4-Methoxybenzaldehyd (2d): 1) $0.185 \mathrm{~g}$ (1.00 mmol) $1 \mathrm{~b}$ werden mit $0.136 \mathrm{~g}$ (1.00 mmol) $2 \mathrm{~d}$ und $0.034 \mathrm{~g}(0.30 \mathrm{mmol}) \mathrm{Me}_{3} \mathrm{COK}$ in $3 \mathrm{ml} \mathrm{DMF} 4 \mathrm{~h}$ bei $20^{\circ} \mathrm{C}$ gerührt. Es wird nach Methode GC/A aufgearbeitet. Ausb. 52\% 1-Chlor-2-I(4-methoxyphenyl)(trimethylsiloxy)methyl]benzol (3j).

2) $3.69 \mathrm{~g}(20 \mathrm{mmol}) \mathbf{1} \mathrm{b}$ werden mit $2.72 \mathrm{~g}(20 \mathrm{mmol}) 2 \mathrm{~d}$ und $0.672 \mathrm{~g}(6.00 \mathrm{mmol}) \mathrm{Me}_{3} \mathrm{COK}$ in $60 \mathrm{ml} \mathrm{DMF} 4 \mathrm{~h}$ bei $20^{\circ} \mathrm{C}$.gerührt. Aufarbeitung nach Methode A. Nach Destillation des

Chem. Ber. 118 (1985) 
Rückstandes Ausb. $2.45 \mathrm{~g}(38 \%)$ 3j, Sdp. $138^{\circ} \mathrm{C} / 4 \cdot 10^{-2}$ Torr. $-{ }^{1} \mathrm{H}-\mathrm{NMR}\left(\mathrm{CDCl}_{3}\right): \delta=$ 0.07 (s, 9H, $\left.\mathrm{Si}\left(\mathrm{CH}_{3}\right)_{3}\right), 3.72\left(\mathrm{~s}, 3 \mathrm{H}, \mathrm{OCH}_{3}\right), 6.17$ (s, $\left.\mathrm{CH}\right), 6.73-7.82$ (m, 8 aromat. $\mathrm{H}$ ).

$\mathrm{C}_{17} \mathrm{H}_{21} \mathrm{ClO}_{2} \mathrm{Si}(320.9)$ Ber. C $63.63 \mathrm{H} 6.59 \mathrm{Cl} 11.04$ Gef. C $63.77 \mathrm{H} 6.89 \mathrm{Cl} 11.04$

II. Mit aliphatischen Aldehyden

a) Von $1 \mathrm{a}$ mit Acetaldehyd (5a): $\mathrm{Zu} 2.93 \mathrm{~g}(15 \mathrm{mmol}) 1 \mathrm{a}$ und $0.66 \mathrm{~g}(15 \mathrm{mmol}) 5 \mathrm{a}$ läßt man bei $0^{\circ} \mathrm{C}$ unter Rühren die Suspension von $0.278 \mathrm{~g}(1.5 \mathrm{mmol}) \mathrm{Et}_{4} \mathrm{NF} \cdot 2 \mathrm{H}_{2} \mathrm{O}$ in $10 \mathrm{ml}$ HMPT tropfen. Nach 30 min Nachrühren wird nach Methode $\mathrm{C}$ mit $20 \mathrm{ml}$ Wasser aufgearbeitet, das Rohprodukt durch Mitteldrucksäulenchromatographie mit PE/EE (20:1) gereinigt und anschließend destilliert. Ausb. $1.86 \mathrm{~g}$ (74\%) 1-(2-Nitrophenyl)ethanol (6a), Sdp. $162^{\circ} \mathrm{C} / 20$ Torr (Lit. ${ }^{18)} 131-132^{\circ} \mathrm{C} / 3$ Torr). $-{ }^{1} \mathrm{H}-\mathrm{NMR}\left(\mathrm{CDCl}_{3}\right): \delta=1.55\left(\mathrm{~d}, 3 \mathrm{H}, \mathrm{CH}_{3}\right)$, $2.60(\mathrm{~s}, \mathrm{OH}), 5.43(\mathrm{q}, \mathrm{CH}), 7.27-8.03(\mathrm{~m}, 4$ aromat. $\mathrm{H})$.

$\mathrm{C}_{8} \mathrm{H}_{9} \mathrm{NO}_{3}$ (167.2) $\quad$ Ber. C 57.48 H 5.43 N 8.38 Gef. C 57.30 H 5.48 N 8.19

b) Von 1 a mit Butyraldehyd (5b): $2.93 \mathrm{~g}(15 \mathrm{mmol}) 1 \mathrm{a}, 1.08 \mathrm{~g}(15 \mathrm{mmol}) 5 \mathrm{~b}$ und $0.087 \mathrm{~g}$ (1.5 mmol) $\mathrm{KF}$ werden in $10 \mathrm{ml} \mathrm{HMPT} 10 \mathrm{~h}$ bei $80^{\circ} \mathrm{C}$ gerührt. Anschließend wird nach Methode $C$ mit $30 \mathrm{ml}$ Wasser aufgearbeitet, mit saurem wäßr. Methanol hydrolysiert und durch Mitteldrucksäulenchromatographie mit PE/EE (20:1) gereinigt. Ausb. 2.16 g (74\%) 1-(2-Nitrophenyl)-1-butanol $(6 \mathrm{~b})$, Sdp. $118^{\circ} \mathrm{C} / 6 \cdot 10^{-2}$ Torr. $-{ }^{1} \mathrm{H}-\mathrm{NMR}\left(\mathrm{CDCl}_{3}\right) ; \delta=$ $0.77-1.77\left(\mathrm{~m}, 3 \mathrm{H}, \mathrm{CH}_{3}\right), 1.17-2.0\left(\mathrm{~m}, 4 \mathrm{H}, \mathrm{CH}_{2} \mathrm{CH}_{2}\right), 2.50(\mathrm{~s}, \mathrm{OH}), 5.25(\mathrm{t}, \mathrm{CH}), 7.37-8.00$ $(\mathrm{m}, 4$ aromat. $\mathrm{H})$.

\section{$\mathrm{C}_{10} \mathrm{H}_{13} \mathrm{NO}_{3}$ (195.2) $\quad$ Ber. C 61.52 H 6.71 N 7.18 Gef. C 61.48 H 6.59 N 7.10}

c) Von $1 \mathrm{~b}$ mit Acetaldehyd (5a): $\mathrm{Zu} 3.69 \mathrm{~g}$ (20 mmol) $1 \mathrm{~b}$ und $0.88 \mathrm{~g}(20 \mathrm{mmol}) 5 \mathrm{a}$ wird bei $0^{\circ} \mathrm{C}$ unter Rühren die Suspension von $0.371 \mathrm{~g}\left(2.00 \mathrm{mmol}^{\circ} \mathrm{Et}_{4} \mathrm{NF} \cdot 2 \mathrm{H}_{2} \mathrm{O}\right.$ in $10 \mathrm{ml}$ HMPT getropft. Nach Rühren über Nacht bei Raumtemp. wird wie unter a) beschrieben aufgearbeitet. Ausb. $1.09 \mathrm{~g}(36 \%) 1-\left(2\right.$-Chlorphenyl)ethanol $(6 \mathrm{c})$, Sdp. $111^{\circ} \mathrm{C} / 14$ Torr (Lit. ${ }^{19}$ ) Sdp. $94^{\circ} \mathrm{C} / 4$ Torr). $-{ }^{1} \mathrm{H}-\mathrm{NMR}\left(\mathrm{CDCl}_{3}\right): \delta=1.48\left(\mathrm{~d}, 3 \mathrm{H}, \mathrm{CH}_{3}\right), 2.25(\mathrm{~s}, \mathrm{OH}), 5.30(\mathrm{q}, \mathrm{CH})$, $6.93-7.73(\mathrm{~m}, 4$ aromat. $\mathrm{H})$.

$\mathrm{C}_{8} \mathrm{H}_{9} \mathrm{ClO}(156.6) \quad$ Ber. $\mathrm{C} 61.35 \mathrm{H} 5.79 \mathrm{Cl} 22.63$ Gef. C $61.33 \mathrm{H} 6.00 \mathrm{Cl} 22.40$

d) Von 1 b mit Butyraldehyd (5 b): $3.69 \mathrm{~g}(20 \mathrm{mmol}) \mathbf{1 ~ b}, 1.44 \mathrm{~g}(20 \mathrm{mmol}) \mathbf{5 b}$ und $0.116 \mathrm{~g}$ (2.00 mmol) $\mathrm{KF}$ in $10 \mathrm{ml}$ HMPT werden $12 \mathrm{~h}$ bei $80^{\circ} \mathrm{C}$ gerührt. Es wird wie unter b) beschrieben aufgearbeitet. Ausb. $1.70 \mathrm{~g}(46 \%)$ l-(2-Chlorphenyl)-1-butanol (6d), Sdp. $126-128^{\circ} \mathrm{C} / 14$ Torr (Lit. ${ }^{20)} 118-120^{\circ} \mathrm{C} / 10$ Torr). $-{ }^{1} \mathrm{H}-\mathrm{NMR}\left(\mathrm{CDCl}_{3}\right): \delta=0.67-1.13$ (m, 3H, $\left.\mathrm{CH}_{3}\right), 1.13-2.00\left(\mathrm{~m}, 4 \mathrm{H}, \mathrm{CH}_{2} \mathrm{CH}_{2}\right), 2.08(\mathrm{~s}, \mathrm{OH}), 5.13(\mathrm{t}, \mathrm{CH}), 7.10-7.65(\mathrm{~m}$, 4 aromat. $\mathrm{H})$.

$\mathrm{C}_{10} \mathrm{H}_{13} \mathrm{ClO}(184.7) \quad$ Ber. $\mathrm{C} 65.04 \quad \mathrm{H} 7.09 \mathrm{Cl} 19.19$ Gef. C $64.88 \quad \mathrm{H} 7.00 \mathrm{Cl} 19.19$

III. Mit Ketonen (7)

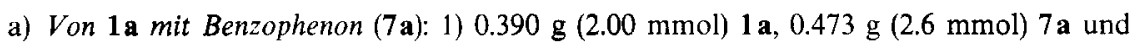
$0.012 \mathrm{~g}(0.20 \mathrm{mmol}) \mathrm{KF}$ in $1.5 \mathrm{ml} \mathrm{HMPT}$ werden bei $80^{\circ} \mathrm{C}$ gerührt. Es wird nach Methode GC/B aufgearbeitet. Ausb. nach $24 \mathrm{~h}$ (ca. 70\% Umsatz) 37\% und nach $48 \mathrm{~h}(100 \% \mathrm{Umsatz})$ $52 \%$ (2-Nitrophenyl)diphenyl (trimethylsiloxy)methan (8a).

2) $2.93 \mathrm{~g}(15 \mathrm{mmol}) 1 \mathrm{a}, 2.73 \mathrm{~g}(15 \mathrm{mmol}) 7 \mathrm{a}$ und $0.168 \mathrm{~g} \mathrm{(1.5} \mathrm{mmol)} \mathrm{Me}_{3} \mathrm{COK}$ werden in $50 \mathrm{ml}$ HMPT $2 \mathrm{~h}$ bei Raumtemp. und dann $3 \mathrm{~h}$ bei $100^{\circ} \mathrm{C}$ gerührt. Nach Aufarbeiten nach Methode C mit $150 \mathrm{ml}$ 4proz. Salzsäure wird das Rohprodukt über eine Kiesclgclsäule mit PE/EE (10:1) gereinigt und der nach Entfernen des Lösungsmittels erhaltene Feststoff aus PE/EE (10:1) umkristallisiert. Ausb. $3.45 \mathrm{~g}(61 \%)$ 8a. $-{ }^{1} \mathrm{H}-\mathrm{NMR}\left(\mathrm{CDCl}_{3}\right): \delta=0.17$ (s, $\left.9 \mathrm{H}, \mathrm{Si}\left(\mathrm{CH}_{3}\right)_{3}\right), 7.30-7.67(\mathrm{~m}, 14$ aromat. $\mathrm{H})$.

$\mathrm{C}_{22} \mathrm{H}_{23} \mathrm{NO}_{3} \mathrm{Si}(377.5) \quad$ Ber. $\mathrm{C} 69.99 \mathrm{H} 6.14$ N 3.71 Gef. C. $70.00 \mathrm{H} 6.00$ N 4.06

Chem. Ber. 118 (1985) 
b) Von $1 \mathrm{a}$ mil Aceton (7b): 1) $0.195 \mathrm{~g}(1.00 \mathrm{mmol}) 1 \mathrm{a}$ und $0.183 \mathrm{~g} \mathrm{(3.15} \mathrm{mmol)} 7 \mathrm{~b}$ in $3 \mathrm{ml}$ HMPT werden mit $0.022 \mathrm{~g}(0.20 \mathrm{mmol}) \mathrm{Me}_{3} \mathrm{COK}$ bei $20^{\circ} \mathrm{C}$ bis zum vollständigen Umsatz gerührt. Aufarbeiten nach Methode GC/B liefert 62\% 2-(2-Nitrophenyl)-2-propanol (9b).

2) $\mathrm{Zu} 2.93 \mathrm{~g}(15 \mathrm{mmol}) 1 \mathrm{a}$ und $2.61 \mathrm{~g}(45 \mathrm{mmol}) 7 \mathrm{~b}$ in $50 \mathrm{ml}$ HMPT werden bei Raumtemp. portionsweise $0.168 \mathrm{~g}(1.5 \mathrm{mmol}) \mathrm{Me}_{3} \mathrm{COK}$ gegeben. Nach $1 \mathrm{~h}$ Rühren bei Raumtemp. wird nach Methode C mit $150 \mathrm{ml}$ 4proz. Salzsäure aufgearbeitet, das Rohprodukt über eine Kieselgelsäule zunächst mit PE/EE (20:1) von Verunreinigungen befreit, dann mit reinem EE eluiert. Ausb. $1.52 \mathrm{~g} \mathrm{(56 \% )} \mathrm{9b,} \mathrm{Schmp.} 85-87^{\circ} \mathrm{C}$ (Lit. ${ }^{21)} \mathrm{Sdp} .142-144^{\circ} \mathrm{C} / 0.1$ Torr). ${ }^{1} \mathrm{H}-\mathrm{NMR}\left(\mathrm{CDCl}_{3}\right): \delta=1.70\left(\mathrm{~s}, 3 \mathrm{H}, \mathrm{CH}_{3}\right), 2.27(\mathrm{~s}, \mathrm{OH}), 7.37-7.63$ (m, 4 aromat. $\mathrm{H}$ ),

\section{$\mathrm{C}_{9} \mathrm{H}_{11} \mathrm{NO}_{3}$ (181.2) Ber. C 59.66 H 6.12 N 7.73 Gef. C 59.57 H 6.11 N 7.71}

c) Von $1 \mathrm{a}$ mit Acetophenon (7c): 1) $2.93 \mathrm{~g}(15 \mathrm{mmol}) 1 \mathrm{a}, 1.80 \mathrm{~g}(15 \mathrm{mmol}) 7 \mathrm{c}$ und $0.278 \mathrm{~g}$ (1.5 mmol) $\mathrm{Et}_{4} \mathrm{NF} \cdot 2 \mathrm{H}_{2} \mathrm{O}$ werden in $50 \mathrm{ml} \mathrm{HMPT} 1 \mathrm{~h}$ bei Raumtemp. und anschließend $12 \mathrm{~h}$ bei $60^{\circ} \mathrm{C}$ gerührt. Nach Aufarbeiten nach Methode B mit $150 \mathrm{ml} 4$ proz. Salzsäure wird das Rohprodukt zunächst über eine Kieselgelsäule mit PE/EE (50:1) und anschließend durch Mitteldrucksäulenchromatographie mit PE/EE (50:1) gereinigt. Ausb. 0.62 g (13\%) 1-(2Nitrophenyl)-1-phenyl-1-(trimethylsiloxy) ethan (8c), Schmp. 79-81 ${ }^{\circ} \mathrm{C} . \quad-{ }^{1} \mathrm{H}-\mathrm{NMR}$ $\left(\mathrm{CDCl}_{3}\right): \delta=0.08\left(\mathrm{~s}, 9 \mathrm{H}, \mathrm{Si}\left(\mathrm{CH}_{3}\right)_{3}\right), 2.18\left(\mathrm{~s}, 3 \mathrm{H}, \mathrm{CH}_{3}\right), 7.08-7.68(\mathrm{~m}, 9$ aromat. $\mathrm{H})$.

\section{$\mathrm{C}_{17} \mathrm{H}_{21} \mathrm{NO}_{3} \mathrm{Si}(315.4)$ Ber. C 64.72 H 6.71 N 4.44 Gef. C 64.94 H 6.61 N 4.48}

2) Jeweils $0.195 \mathrm{~g}(1.00 \mathrm{mmol}) 1 \mathrm{a}$ und $0.12 \mathrm{~g}(1.0 \mathrm{mmol}) 7 \mathrm{c}$ werden in $3 \mathrm{ml}$ Lösungsmittel mit $0.25 \mathrm{mmol}$ Katalysator umgesetzt.

\begin{tabular}{|c|c|c|c|c|c|c|}
\hline \multirow{2}{*}{$\begin{array}{l}\text { Katalysator } \\
\text { (mg) }\end{array}$} & \multirow{2}{*}{$\begin{array}{l}\text { Lösungs- } \\
\text { mittel }\end{array}$} & \multicolumn{2}{|c|}{ Reaktions- } & \multirow{2}{*}{$\begin{array}{c}\text { Aufarbeitung } \\
\text { nach } \\
\text { Methode }\end{array}$} & \multicolumn{2}{|c|}{ Ausb. $\%$} \\
\hline & & $\begin{array}{l}\text { zeit } \\
\text { (h) }\end{array}$ & $\begin{array}{c}\text { temp. } \\
\left({ }^{\circ} \mathrm{C}\right)\end{array}$ & & $8 \mathrm{c}$ & $\begin{array}{l}\text { Nitro- } \\
\text { benzol }\end{array}$ \\
\hline $\mathrm{Me}_{3} \mathrm{COK}(28)$ & HMPT & 2 & 20 & $\mathrm{GC} / \mathrm{B}$ & 7 & 87 \\
\hline $\mathrm{Et}_{4} \mathrm{NF}-2 \mathrm{H}_{2} \mathrm{O}(46)$ & HMPT & 1 & 20 & $\mathrm{GC} / \mathrm{B}$ & 9 & 88 \\
\hline $\mathrm{Et}_{4} \mathrm{NF} \cdot 2 \mathrm{H}_{2} \mathrm{O}(46)$ & DMF & 2 & -20 & $\mathrm{GC} / \mathrm{B}$ & 5 & 92 \\
\hline $\mathrm{Et}_{4} \mathrm{NF} \cdot 2 \mathrm{H}_{2} \mathrm{O}(46)$ & DMF & 2 & -20 & $\mathrm{GC} / \mathrm{A}$ & 5 & 90 \\
\hline $\mathrm{KF}(15)$ & HMPT & 17 & 100 & $\mathrm{GC} / \mathrm{A}$ & 9 & 82 \\
\hline $\mathrm{KF}(15)$ & HMPT & 17 & 100 & $\mathrm{GC} / \mathrm{B}$ & 9 & 85 \\
\hline CsF (38) & HMPT & 24 & 20 & $\mathrm{GC} / \mathrm{B}$ & 11 & 68 \\
\hline
\end{tabular}

Nach wäßriger Aufarbeitung wurde Acetophenon (7c) gaschromatographisch mit ca. $80 \%$ Ausb. nachgewiesen.

d) Von 1 a mit Cyclohexanon (7d): $2.93 \mathrm{~g}(15 \mathrm{mmol}) \mathbf{1 a}, 1.47 \mathrm{~g}(15 \mathrm{mmol}) \mathbf{7 d}$ und $0.087 \mathrm{~g}$ (1.5 mmol) KF werden in $50 \mathrm{ml}$ HMPT $48 \mathrm{~h}$ bei $80^{\circ} \mathrm{C}$ gerührt. Nach Aufarbeiten nach Methode C mit $150 \mathrm{ml}$ 4proz. Salzsäure wird das Rohprodukt zunächst über eine Kieselgelsäule mit PE/EE (9:1), dann durch Mitteldrucksäulenchromatographie mit $\mathrm{PE} / \mathrm{EE}$ (50:1) gereinigt und anschließend destilliert. Ausb. $0.75 \mathrm{~g} \mathrm{(17 \% )} \mathrm{1-(2-Nitrophenyl)-1-(tri-}$ methylsiloxy)cyclohexan (8d). $-{ }^{1} \mathrm{H}-\mathrm{NMR}\left(\mathrm{CDCl}_{3}\right): \delta=0.03\left(\mathrm{~s}, 9 \mathrm{H}, \mathrm{Si}\left(\mathrm{CH}_{3}\right)_{3}\right), 1.42-2.43$ $\left(\mathrm{m}, 10 \mathrm{H},\left[\mathrm{CH}_{2}\right]_{5}\right), 7.33-7.93$ (m, 4 aromat. $\left.\mathrm{H}\right)$.

$$
\mathrm{C}_{15} \mathrm{H}_{23} \mathrm{NO}_{3} \mathrm{Si} \text { (293.4) Ber. C } 61.39 \text { H } 7.90 \quad \mathrm{~N} 4.77 \text { Gef. C } 61.30 \text { H } 7.88 \quad \mathrm{~N} 4.90
$$

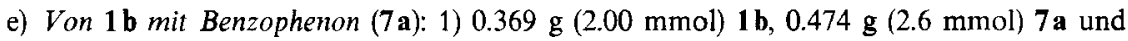
$0.012 \mathrm{~g}(0.20 \mathrm{mmol}) \mathrm{KF}$ werden in $1.5 \mathrm{ml} \mathrm{HMPT}$ bei $80^{\circ} \mathrm{C}$ gerührt. Es wird nach Methode GC/B aufgearbeitet. Ausb. nach 24 h $9 \%$ und nach 39 h $39 \%$ (2-Chlorphenyl)diphenyl trimethylsiloxy)methan (8e). 
2) $\mathrm{Zu} 2.77 \mathrm{~g}(15 \mathrm{mmol}) 1 \mathrm{~b}$ und $2.73 \mathrm{~g}(15 \mathrm{mmol}) 7 \mathrm{a}$ in $50 \mathrm{ml} \mathrm{HMPT}$ werden portionsweise unter Rühren bei Raumtemp. $0.589 \mathrm{~g}(5.25 \mathrm{mmol}) \mathrm{Me}_{3} \mathrm{COK}$ gegeben. Nach $2 \mathrm{~h}$ Rühren bei Raumtemp. wird nach Methode C mit $150 \mathrm{ml}$ 4proz. Salzsäure aufgearbeitet, das Rohprodukt über eine Kieselgelsäule mit PE/EE (10:1) eluiert und durch Mitteldrucksäulenchromatographie mit PE/EE (50:1) gereinigt. Ausb. $2.54 \mathrm{~g} \mathrm{(46 \% )} \mathrm{8e,} \mathrm{Schmp.} 54-56.5^{\circ} \mathrm{C}$ - - ${ }^{1} \mathrm{H}-$ NMR $\left(\mathrm{CDCl}_{3}\right): \delta=0.12\left(\mathrm{~s}, 9 \mathrm{H}, \mathrm{Si}\left(\mathrm{CH}_{3}\right)_{3}\right), 7.33-8.12$ (m, 14 aromat. $\left.\mathrm{H}\right)$.

\section{$\mathrm{C}_{22} \mathrm{H}_{23} \mathrm{ClOSi}(367.0)$ Ber. C $72.00 \mathrm{H} 6.31 \mathrm{Cl} 9.66$ Gef. C $72.02 \mathrm{H} 6.51 \mathrm{Cl} 9.74$}

f) Von $1 \mathrm{~b}$ mit Aceton (7b): $3.69 \mathrm{~g}(20 \mathrm{mmol}) 1 \mathrm{~b}, 2.32 \mathrm{~g} \mathrm{(40} \mathrm{mmol)} 7 \mathrm{~b}$ und $1.112 \mathrm{~g}(6.0$ mmol) $\mathrm{Et}_{4} \mathrm{NF} \cdot 2 \mathrm{H}_{2} \mathrm{O}$ werden in $50 \mathrm{ml} \mathrm{HMPT} 1 \mathrm{~h}$ bei $20^{\circ} \mathrm{C}$ gerührt. Nach Methode $\mathrm{C}$ wird mit $150 \mathrm{ml}$ 4proz. Salzsäure aufgearbeitet. Das Rohprodukt wird zunächst über eine Kieselgelsäule mit PE/EE (25:1), dann durch Mitteldrucksäulenchromatographie mit PE/EE (25:1) gereinigt und anschließend destilliert. Ausb. 0.95 g (28\%) 2-(2-Chlorphenyl)-2-propanol (9f), Sdp. $110-112^{\circ} \mathrm{C} / 18$ Torr (Lit. ${ }^{22)} 108-111^{\circ} \mathrm{C} / 12$ Torr).

g)Von $1 \mathrm{~b}$ mit Acetophenon (7c): $3.69 \mathrm{~g}(20 \mathrm{mmol}) 1 \mathrm{~b}, 2.40 \mathrm{~g}(20 \mathrm{mmol}) 7 \mathrm{c}$ und $1.48 \mathrm{~g}(8$ mmol) $\mathrm{Et}_{4} \mathrm{NF} \cdot 2 \mathrm{H}_{2} \mathrm{O}$ werden in $50 \mathrm{ml} \mathrm{HMPT} 2 \mathrm{~h}$ bei Raumtemp. gerührt. Nach Methode $\mathrm{C}$ wird mit $150 \mathrm{ml}$ 4proz. Salzsäure aufgearbeitet. Das Rohprodukt wird zunächst über eine Kieselgelsäule mit PE/EE (25:1) und anschließend durch Mitteldrucksäulenchromatographie mit PE/EE (25:1) gereinigt. Ausb. $0.37 \mathrm{~g}$ (8\%) 1-(2-Chlorphenyl-)-1-phenylethanol (9g). $-{ }^{1} \mathrm{H}-\mathrm{NMR}\left(\mathrm{CDCl}_{3}\right): \delta=1.97\left(\mathrm{~s}, 3 \mathrm{H}, \mathrm{CH}_{3}\right), 3.33(\mathrm{~s}, \mathrm{OH}), 7.27-8.13(\mathrm{~m}, 9$ aromat. H).

$\mathrm{C}_{14} \mathrm{H}_{13} \mathrm{ClO}(232.7)$ Ber. C 72.25 H $5.63 \mathrm{Cl} 15.23$ Gef. C 72.15 H $5.74 \mathrm{Cl} 15.26$

IV. Mit Acylierungsmitteln

a) Von 1 a mit Benzoylfluorid (10a): 1) $0.391 \mathrm{~g}(2.00 \mathrm{mmol}) 1 \mathrm{a}, 0.248 \mathrm{~g}(2.00 \mathrm{mmol}) 10 \mathrm{a}$ und $0.029 \mathrm{~g}(0.50 \mathrm{mmol}) \mathrm{KF}$ werden in $1.5 \mathrm{ml} \mathrm{HMPT} 2 \mathrm{~h}$ bei $100^{\circ} \mathrm{C}$ gerührt. Nach Methode GC/B gewinnt man 59\% 2-Nitrobenzophenon (12a).

2) $2.93 \mathrm{~g}(15 \mathrm{mmol}) 1 \mathrm{a}, 1.86 \mathrm{~g}(15 \mathrm{mmol}) \mathbf{1 0 a}$ und $0.174 \mathrm{~g}(3.0 \mathrm{mmol}) \mathrm{KF}$ werden in 10 ml HMPT $6 \mathrm{~h}$ bei $80^{\circ} \mathrm{C}$ gerührt. Man arbeitet nach Methode C mit $30 \mathrm{ml}$ Wasser auf. Das Rohprodukt wird über eine Kieselgelsäule mit PE/EE (20:1) gereinigt, der erhaltene Feststoff aus Ethanol umkristallisiert. Ausb. $2.01 \mathrm{~g}(59 \%)$ 12a, Schmp. $105-106^{\circ} \mathrm{C}$ (Lit. ${ }^{23 a)} 105^{\circ} \mathrm{C}$ ).

b) Von $1 \mathrm{~b}$ mit $10 \mathrm{a}: 2.77 \mathrm{~g}(15 \mathrm{mmol}) 1 \mathrm{~b}, 1.86 \mathrm{~g}(15 \mathrm{mmol}) \mathbf{1 0 a}$ und $0.174 \mathrm{~g}(3.0 \mathrm{mmol})$ $\mathrm{KF}$ werden in $10 \mathrm{ml} \mathrm{HMPT} 96 \mathrm{~h}$ bei $100^{\circ} \mathrm{C}$ gerührt und wie vorstehend unter 2) beschrieben aufgearbeitet. Ausb. $1.43 \mathrm{~g}(44 \%)$ 2-Chlorbenzophenon (12b), Schmp. $45.5^{\circ} \mathrm{C}$ (Lit. ${ }^{23 \mathrm{~b})}$ $52-56^{\circ} \mathrm{C}$ ).

c) Von 1 a mit Acetylfluorid: $2.93 \mathrm{~g}(15 \mathrm{mmol}) 1 \mathrm{a}$ werden mit $1.86 \mathrm{~g}(30 \mathrm{mmol})$ Acetylfluorid und $0.228 \mathrm{~g}$ (1.5 mmol) Cäsiumfluorid in $10 \mathrm{ml}$ DMF $14 \mathrm{~d}$ bei Raumtemp. gerührt. Es wird nach Methode GC/B aufgearbeitet. Neben Nitrobenzol als Hauptprodukt werden 10\% Methyl(2-nitrophenyl)keton gaschromatographisch durch Koinjektion nachgewiesen.

d) Von 1 a mit Benzoesäureanhydrid (10 b): 1) $0.195 \mathrm{~g}$ (1.00 mmol) 1 a werden mit $0.226 \mathrm{~g}$ (1.00 mmol) $10 \mathrm{~b}$ und $0.056 \mathrm{~g}(0.50 \mathrm{mmol})$ Kaliumbenzoat bzw. $0.058 \mathrm{~g}(1.0 \mathrm{mmol}) \mathrm{KF}$ in 1 ml HMPT $24 \mathrm{~h}$ bei $100^{\circ} \mathrm{C}$ gerührt. Nach Methode GC/A wird aufgearbeitet. Ausb. mit Kaliumbenzoat $32 \% 12 \mathrm{a}$, mit KF $24 \% 12 \mathrm{a}$.

2) $2.93 \mathrm{~g}(15 \mathrm{mmol}) 1 \mathrm{a}, 3.39 \mathrm{~g}(15 \mathrm{mmol}) 10 \mathrm{~b}$ und $0.556 \mathrm{~g}(3.0 \mathrm{mmol}) \mathrm{Et}_{4} \mathrm{NF} \cdot 2 \mathrm{H}_{2} \mathrm{O}$ werden in $10 \mathrm{ml} \mathrm{HMPT} 24 \mathrm{~h}$ bei $100^{\circ} \mathrm{C}$ gerührt. Aufarbeitung nach Methode C. Das Rohprodukt wird über eine Kieselgelsäule mit PE/EE $(10: 1)$ vorgereinigt und in Ether aufgenommen. Die etherische Lösung wird mit verdünnter wäßr. Natronlauge ausgeschüttelt (zur Entfernung von Benzoesäure) und der nach Entfernen des Ethers verbleibende Rückstand aus Ethanol umkristallisiert. Ausb. $1.06 \mathrm{~g}(31 \%)$ 12a, Schmp. $105^{\circ} \mathrm{C}$.

Chem. Ber. 118 (1985) 
Umsetzung von 1 a mit 2-Nitrobenzophenon (12a): $1.95 \mathrm{~g}(10 \mathrm{mmol}) 1 \mathrm{a}, 2.27 \mathrm{~g}(10 \mathrm{mmol})$ $12 \mathrm{a}$ und $0.116 \mathrm{~g}(2.0 \mathrm{mmol}) \mathrm{KF}$ werden in $10 \mathrm{ml} \mathrm{HMPT} 24 \mathrm{~h}$ bei $100^{\circ} \mathrm{C}$ gerührt. Der nach Aufarbeitungs-Methode $C$ erhaltene Rückstand wird über eine Kieselgelsäule mit PE/EE (10:1) vorgereinigt und das so erhaltene Produktgemisch durch Mitteldrucksäulenchromatographie mit PE/EE (10:1) aufgetrennt. 4. Fraktion: Ausb. $0.17 \mathrm{~g}(6 \%)$ 2-(2-Nitrophenyl)benzophenon (13b). - IR (flüssig): $1660(\mathrm{C}=\mathrm{O}), 1355$ und $1530 \mathrm{~cm}^{-1}\left(\mathrm{NO}_{2}\right)$.

$$
\mathrm{C}_{19} \mathrm{H}_{13} \mathrm{NO}_{3} \text { (303.3) Ber. C } 75.24 \text { H } 4.32 \text { N } 4.62 \text { Gef. C } 74.10 \text { H } 4.32 \text { N } 4.23
$$

6. Fraktion: $0.20 \mathrm{~g}(6 \%)$ Bis(2-nitrophenyl) phenylmethanol (13a), Schmp. $199-201^{\circ} \mathrm{C}$. IR (KBr): $3505(\mathrm{OH}), 1525,1545$ und 1355, $1380 \mathrm{~cm}^{-1}\left(\mathrm{NO}_{2}\right)$.

$\mathrm{C}_{19} \mathrm{H}_{14} \mathrm{~N}_{2} \mathrm{O}_{5}(350.3)$ Ber. C 65.14 H 4.03 N 7.99 Gef. C 65.27 H 4.03 N 7.89

\section{Mit Formylierungsmitteln}

Von 1 a mit DMF: 1) Zu $0.195 \mathrm{~g}(1.00 \mathrm{mmol}) 1 \mathrm{a}$ in $3 \mathrm{ml}$ DMF werden in 5 Portionen bei Raumtemp. unter Rühren insgesamt $0.022 \mathrm{~g}(0.20 \mathrm{mmol}) \mathrm{Me}_{3} \mathrm{COK}$ gegeben. Nach jeder Zugabe wird 30 min bei Raumtemp. gerührt und anschließend eine Probe nach Methode GC/B aufgearbeitet.

\begin{tabular}{|c|c|c|c|c|c|}
\hline \multirow{2}{*}{ Produkte } & \multicolumn{5}{|c|}{ Ausb. in $\%$ nach der } \\
\hline & 1. & 2. & $\begin{array}{c}3 . \\
\text { Zugabe }\end{array}$ & 4. & 5. \\
\hline $1 \mathrm{a}$ & 100 & 25 & - & - & - \\
\hline 2-Nitrobenzaldehyd (14) & & 46 & 45 & 42 & 2 \\
\hline Nitrobenzol & & 29 & 32 & 34 & 63 \\
\hline
\end{tabular}

2) $\mathrm{Zu} 1.95 \mathrm{~g}$ (10 mmol) $1 \mathrm{a}$ in $30 \mathrm{ml}$ DMF werden in drei Portionen unter Rühren bei Raumtemp. insgesamt $0.137 \mathrm{~g}(1.22 \mathrm{mmol}) \mathrm{Me}_{3} \mathrm{COK}$ gegeben, nach jeder $\mathrm{Me}_{3} \mathrm{COK}-\mathrm{Zugabe}$ wird 30 min gerührt und anschließend eine Probe nach GC/B aufgearbeitet. Produktverteilung (FID-\%): 47\% 14, 25\% 1 a und 28\% Nitrobenzol. Das Reaktionsgemisch wird nach Methode C mit $100 \mathrm{ml}$ 2proz. Salzsäure aufgearbeitet, das erhaltene Rohprodukt in $10 \mathrm{ml}$ Ethanol gelöst und die Lösung zu $2.0 \mathrm{~g}$ 2,4-Dinitrophenylhydrazin in $50 \mathrm{ml} 30$ proz. Perchlorsäure getropft. Der gelbe Niederschlag wird abgesaugt, das Filtrat mit $50 \mathrm{ml}$ Wasser verdünnt und der so erhaltene Niederschlag ebenfalls abgesaugt. Nach Umkristallisation der Niederschläge aus Ethanol Ausb. $1.14 \mathrm{~g}(42 \%)$ 2-Nitrobenzaldehyd-2,4-dinitrophenylhydrazon, Schmp. $250^{\circ} \mathrm{C}$ (Zers.) (Lit. ${ }^{24)} 247-248^{\circ} \mathrm{C}$ ).

\section{Mit Kohlendioxid}

a) Von $1 \mathrm{a}: 1)$ Jeweils $0.98 \mathrm{~g}$ (5.0 mmol) $1 \mathrm{a}$ und $0.76 \mathrm{~g}$ (5.0 mmol) CsF bzw. $0.29 \mathrm{~g} \mathrm{(5.0}$ mmol) KF werden in $5 \mathrm{ml}$ HMPT im Autoklaven bei 50 atm $\mathrm{CO}_{2} 24 \mathrm{~h}$ bei $80^{\circ} \mathrm{C}$ gerührt. Nach Einrühren des Reaktionsgemisches in verdünnte Natronlauge wird mit Chloroform ausgeschüttelt, die wäßr. alkalische Phase abgetrennt, mit Salzsäure angesäuert und der ausgefallene farblose Niederschlag ausgeethert. Ausb. mit CsF $0.60 \mathrm{~g}(72 \%)$ und mit KF $0.64 \mathrm{~g} \mathrm{(76 \% )} \mathrm{2-Nitrobenzoesäure} \mathrm{(17a),} \mathrm{Schmp.} 147^{\circ} \mathrm{C}$ (Lit. ${ }^{23 \mathrm{c})} 147-148^{\circ} \mathrm{C}$ ).

2) Aus $1.95 \mathrm{~g}(10 \mathrm{mmol}) 1 \mathrm{a}$ und $0.152 \mathrm{~g}(1.0 \mathrm{mmol}) \mathrm{CsF}$ in $10 \mathrm{ml}$ HMPT wie vorstehend beschrieben. Ausb. $0.17 \mathrm{~g}(10 \%) 17 \mathrm{a}$.

b) Von $1 \mathrm{~b}: 1.84 \mathrm{~g}$ (10 mmol) $\mathbf{1 b}$ werden mit $0.58 \mathrm{~g}$ (10 mmol) KF in $10 \mathrm{ml}$ HMPT wie vorstehend beschrieben umgesetzt und aufgcarbeitet. Ausb. $0.90 \mathrm{~g}(58 \%) 2$-Chlorbenzoesäure (17b), Schmp. $142^{\circ} \mathrm{C}$ (Lit. ${ }^{23 d)} 142^{\circ} \mathrm{C}$ ).

Chem. Ber. 118 (1985) 
1) Zur elektrophilen Aromatensubstitution, 28; 27. Mitteil.: F. Effenberger und W. Spiegler, Chem. Ber. 118, 3872 (1985), vorstehend.

2) W. Spiegler, Teil der Dissertation, Univ. Stuttgart 1980.

3) F. Effenberger, Angew. Chem. 92, 147 (1980); Angew. Chem., Int. Ed. Engl. 19, 151 (1980).

4) 4aj $P$. Buck und G. Köbrich, Tetrahedron Lett. 1967, 1563. - ${ }^{4 b)} M$. S. Kharasch und $O$. Reinmuth, Grignard Reactions of Nonmetallic Substances, Prentice Hall Inc., New York 1954.

5) 5a) H. Gilman und R. D. Gorsich, J. Am. Chem. Soc. 79, 2625 (1957). - ${ }^{5 b)}$ R. W. Hofmann, Dehydrobenzene and Cycloalkynes, Academic Press, New York and London 1967. ${ }_{5 c)}$ O. M. Nefedov, A. I. D'yachenko und A. K. Prokof ev, Russ. Chem. Rev. 46, 941 (1977).

6) 6a) G. Köbrich und P. Buck, Chem. Ber. 103, 1412 (1970). - W. E. Parham und Ch. K. Bradsher, Acc. Chem. Res. 15, 300 (1982).

7) Y. Ogata und A. Kawasaki in S. Patai, The Chemistry of the Carbonyl Group, Vol. 2, S. $1 \mathrm{ff}$., Interscience Publishers, London 1970.

${ }^{8)} S$. Forsén und M. Nilsson in Lit.7), S. $157 \mathrm{ff}$.

9) 9a) B. A. Gostevskii, O. A. Kruglaya und $N$. S. Vyazankin, Izv. Akad. Nauk SSSR, Ser. Khim. 1978, 2425 [Chem. Abstr. 90, 55012 u (1979)]. - ${ }^{96)}$ B. A. Gostevksii, O. A. Kruglaya, A. I. Albanov und N. S. Vyazankin, J. Organomet. Chem. 187, 157 (1980).

10) J. Cuomo und R. A. Olofson, J. Org. Chem. 44, 1016 (1979).

${ }^{11)}$ iia) L. Bouvaoult, Bull. Soc. Chim. Fr. 31, $1322(1904) .{ }^{116)}$ L. I. Smith und M. Bayliss, J. Org. Chem. 6, 437 (1941).

12) R. J. Mills und V. Snieckus, Tetrahedron Lett. 25, 483 (1984).

13) R. Kaiser, Chromatographie in der Gasphase, S. 209, Bibliographisches Institut, Mannheim 1965.

14) R. Glatz, Dissertation Univ., Stuttgart 1977.

${ }^{15)}$ A. J. Speziale, K.W. Ratts und D.E. Bissing, Org. Synth., Coll. Vol. V, 361 (1973).

${ }^{16)}$ H. Kobler, R. Minz, G. Al Gasser und G. Simchen, Liebigs Ann. Chem. 1978, 1937.

17) E. Cohen, Rec. Trav. Chim. Pays-Bas 38, 117 (1919).

18) A. H. Ford-Moore und H. N. Rydon, J. Chem. Soc. 1946, 679.

19) C. G. Overberger, J. H. Saunders, R. E. Allen und R. Gander, Org. Synth., Coll. Vol. III, $200(1955)$.

20) D. J. Cram, J. Am. Chem. Soc. 74, 2152 (1952).

21) A. R. Chandhuri und T. N. Ghosh, J. Indian Chem. Soc. 28, 563 (1951).

${ }^{22)}$ C. K. Bradsher und E. S. Smith, J. Am. Chem. Soc. 65, 1643 (1943).

23) 23 a) $R$. C. Weast, Handbook of Chemistry and Physics, 63. Ed., C-163, The Chemical Rubber Co., Cleveland, Ohio 1982-1983. - ${ }^{23 b)}$ Lit. $^{23 a)}$, C-160. - ${ }^{23 c)}$ Lit. $^{23 a)}$, C-156. 23d) Lit. $^{23 a)}$, C-151.

24) M. Ragno, Gazz. Chim. Ital. 75, 175, 183 (1945). 